\title{
Classical typicality of the canonical distribution
}

\author{
A. R. Plastino ${ }^{1,2,3(a)}$ and A. Daffertshofer ${ }^{4(\mathrm{~b})}$ \\ ${ }^{1}$ Instituto Carlos I de Física Teórica y Computacional, University of Granada - Granada, Spain, EU \\ ${ }^{2}$ National University La Plata, CREG-UNLP-CONICET - C.C. 727, 1900 La Plata, Argentina \\ ${ }^{3}$ Physics Department, University of Pretoria - Pretoria 0002, South Africa \\ ${ }^{4}$ Research Institute MOVE, Faculty of Human Movement Sciences, VU University Amsterdam \\ van der Boechorststraat 9, 1081 BT, Amsterdam, The Netherlands, EU
}

received 9 July 2008; accepted in final form 24 September 2008

published online 30 October 2008

PACS 05.20.Gg - Classical ensemble theory

\begin{abstract}
We consider the typicality of the canonical ensemble's probability distribution from a classical perspective, resuming recent discussions on quantum-mechanical aspects of canonical typicality. In the conventional derivation of the classical canonical distribution for a system $S$ that is weakly coupled to a heat bath $B$, it is assumed that the composite $S+B$ is represented by the microcanonical ensemble i.e., by a uniform probability distribution on an energy shell of the composite $S+B$. Here we show that for a very large heat bath almost all probability distributions defined on this energy shell behave according to the microcanonical ensemble, yielding a marginal probability distribution for $S$ of the canonical form. Consequently, the classical canonical distribution can be regarded as much more "typical" than suggested by the standard derivation.
\end{abstract}

Copyright (c) EPLA, 2008

When deriving the canonical ensemble's probability distribution corresponding to a system $S$, which is weakly coupled (and in thermal equilibrium) with a heat bath $B$, one usually assumes that the composite $S+B$ is described by the microcanonical ensemble distribution $[1,2]$. The composite $S+B$ is represented by a uniform probability distribution in an energy shell corresponding to states with energies within an interval $[E-\Delta, E+\Delta]$ with $\Delta \ll E$. This derivation is valid in classical and quantummechanical scenarios alike. More recently, however, it has been pointed out that the quantum-mechanical case allows to weaken assumptions substantially when deriving the canonical distribution. For the majority of pure states of the composite $S+B$ belonging to the subspace associated with the aforementioned energy interval, the reduced state of the system $S$ is represented by a canonical ensemble density matrix [3,4]. This result has attracted considerable attention in recent years [5-13]. A detailed and instructive analysis of its application to several quantum model systems can be found in ref. [4]. The aim of the present letter is to show that this robust characteristic of the quantum-canonical distribution has a classical counterpart. Based on a purely classical

\footnotetext{
(a) E-mail: arplastino@maple.up.ac.za

(b) E-mail: marlow@fbw.vu.nl
}

probabilistic line of reasoning, we argue that for "typical" probability distributions defined in an energy shell, i.e., not necessarily microcanonical distributions, the marginal probability distribution corresponding to the system $S$ exhibits Gibbs' canonical form.

There are numerous motivations for exploring the robustness of the canonical distribution from a classical point of view. First, there are many important classical settings for which it is important to asses the typicality of the corresponding canonical distribution. For example consider a classical, microcanonical molecular dynamics simulation of an $N$-particle system. As the (Hamiltonian) evolution proceeds, the system moves along a trajectory contained within a hyper-surface of constant energy. When concentrating on a small subsystem of the $N$-particle system, for instance just one single molecule, it is found that the time averages of the corresponding dynamical quantities coincide with the averages computed from an appropriate canonical distribution of that subsystem. This can be explained assuming that the full $N$-particle system surveys the constant-energy hyper-surface uniformly, so that it is represented by a microcanonical probability distribution. It would be very appealing to weaken the latter assumption, that is, considering the case in which the distribution describing the full system is not strictly microcanonical, while still being able to obtain a canonical 
distribution for the subsystem. Second, the argument for the typicality of the canonical distribution may offer new (speculative) fuel for the epistemic vs. ontic debate on the foundations of quantum mechanics [14]. The classical argument for the robustness of the canonical distribution, when contrasted with its quantum-mechanical counterpart may be regarded as supporting the epistemic interpretation of the quantum wave function, because it illustrates once more the deep similarities that exist between classical probabilities, on the one hand, and probabilities of quantum origin, on the other one [14-16]. Finally, it is worth stressing that most textbooks of statistical mechanics derive the canonical distribution for a given system from the microcanonical distribution associated with the composite "system + bath". It is certainly important to assess the possibility of obtaining the canonical distribution under more general conditions, without the microcanonial assumption.

In our derivation we assume discrete and finite state spaces $\Omega_{S}$ and $\Omega_{B}$ for the system and the bath, respectively. The composite $S+B$ is described by the Cartesian product space $\Omega_{S+B}=\Omega_{S} \times \Omega_{B}$. Notice that these discrete spaces represent classical settings, that is, our state spaces are classical rather than Hilbert spaces, and instead of quantum-mechanical wave functions or density matrices we consider classical probability distributions defined on $\Omega_{S+B}$. We further assume that the interaction term in the total Hamiltonian governing the evolution of $S+B$ is sufficiently small to be neglected. In other words, the total Hamiltonian will be written as a sum $H^{(S+B)}=H^{(S)}+H^{(B)}$ and, consequently, the total energy can be cast into

$$
E^{(S+B)}=E^{(S)}+E^{(B)} .
$$

Finally, let $\omega_{S+B}(E)$ denote an energy shell consisting of all states of $S+B$ with total energy $E^{(S+B)}$ in the interval $[E-\Delta, E+\Delta]$. Then, the microcanonical distribution $p_{k}^{\text {micro }}$ is defined as follows: if the state $k$ does not belong to $\omega_{S+B}(E)$, then we have $p_{k}=0$; however, if the state $k$ belongs to $\omega_{S+B}(E)$, then $p_{k}=1 / N\left[\omega_{S+B}(E)\right]$ holds, where $N\left[\omega_{S+B}(E)\right]$ refers to the total number of states in $\omega_{S+B}(E)$.

The key ingredient in the conventional proof that the marginal probability distribution for $\Omega_{S}$ has a canonical form is that the joint probability distribution on the energy shell $\omega_{S+B}(E)$ complies with the following property: for any subset $A \subset \omega_{S+B}(E)$ with $M$ elements, the total probability $p^{S+B}(A)$ for the state of the composite $S+B$ to be in $A$ is given by

$$
p^{(S+B)}(A)=\frac{M}{N\left[\omega_{S+B}(E)\right]} .
$$

This property, in turn, when combined with (1) can be used to determine the probability of finding the system $S$ in a state $j$ with energy $\varepsilon_{j}$; see, for instance, [1,17]. If the system $S$ is in state $j$, then the energy of the heat bath $B$ must be within the energy interval $\left[E-\varepsilon_{j}-\Delta\right.$, $\left.E-\varepsilon_{j}+\Delta\right]$. The total number of states of $B$ with energies in this range is $2 \Delta g^{(B)}\left(E-\varepsilon_{j}\right)$, where $g^{(B)}(E)$ is the density of states of $B$. Therefore, eq. (2) implies that the probability of finding the system in state $j$ is

$$
p_{j}^{(S)}=\frac{2 \Delta}{N\left[\omega_{S+B}(E)\right]} g^{(B)}\left(E-\epsilon_{j}\right) .
$$

Obviously, the microcanonical distribution, i.e. the uniform probability distribution on $\omega_{S+B}(E)$, complies with (2). As will be shown below, most probability distributions on $\omega_{S+B}(E)$ share the aforementioned property (2) for large $N$.

To simplify notation, we abbreviate $N\left[\omega_{S+B}(E)\right]$ by $N$ when referring to the total number of states in $\omega_{S+B}(E)$. Let $p_{i}$ be the corresponding probabilities associated with the individual states $i=1,2, \ldots, N$ of the energy shell $\omega_{S+B}(E)$. As $0 \leqslant p_{i} \leqslant 1$, we can substitute $p_{i}=x_{i}^{2}$ with $-1 \leqslant x_{i} \leqslant 1$. Therefore, the normalization of the total probability implies

$$
\sum_{i=1}^{N} p_{i}=\sum_{i=1}^{N} x_{i}^{2}=1
$$

In words, the probability distributions correspond to points on $N$-dimensional hyper-sphere in the $\left\{x_{i}\right\}$ space. In order to study the typical behavior of "most" probability distributions defined on the set $\omega_{S+B}(E)$, it is necessary to define a "uniform distribution" over the set of probability distributions $\left\{p_{i}\right\}$. There is an intuitive way to introduce a Riemann metric in this probability space $[18,19]$, characterized by the line element

$$
\mathrm{d} s^{2}=\sum_{i=1}^{N} \frac{1}{2 p_{i}} \mathrm{~d} p_{i}^{2}=\sum_{i=1}^{N} \mathrm{~d} x_{i}^{2} .
$$

This metric, when expressed in terms of the $\left\{x_{i}\right\}$ variables, is the conventional $N$-dimensional Euclidean metric. Using the measure associated with (5), the uniform distribution on the probability space $\left\{p_{i}\right\}$ becomes a uniform distribution (in the standard geometrical sense) on the $N$-dimensional hyper-sphere in $\left\{x_{i}\right\}$ space, which is given by

$$
f\left(x_{1}, x_{2}, \cdots, x_{N}\right)=\frac{2}{S_{N}(1)} \delta\left[\sum_{i=1}^{N} x_{i}^{2}-1\right]
$$

$\delta(x)$ denotes Dirac's delta-distribution and

$$
S_{N}(r)=\frac{2 \pi^{\frac{N}{2}} r^{N-1}}{\Gamma(N / 2)}
$$

stands for the surface area of an $N$-dimensional hypersphere. Note that, in order to have a one-to-one correspondence between the probability distributions $\left\{p_{i}\right\}$ and the points on the $\left\{x_{i}\right\}$ hyper-sphere, one should consider only 
the positive hyper-octant. It is easy to see, however, that the average of any function of the squares $x_{i}^{2}$, on which we build our present argument, adopts the same value on every of the $2^{N}$ hyper-octants; this is also the value one obtains when averaging the function under study over the full hyper-sphere.

Next we investigate the typical behaviour of the quantity

$$
R^{2}=\sum_{i=1}^{M} p_{i}=\sum_{i=1}^{M} x_{i}^{2}
$$

with $1 \leqslant M<N$. To compute the corresponding moments $\mu_{n}^{(M)}=\left\langle R^{2 n}\right\rangle$, we consider the marginal distribution $f^{(M)}\left(x_{1}, \ldots, x_{M}\right)$ which can be cast as

$f^{(M)}=\frac{2}{S_{N}(1)} \int \delta\left[\sum_{i=M+1}^{N} x_{i}^{2}-\left(1-R^{2}\right)\right] \mathrm{d} x_{M+1} \cdots \mathrm{d} x_{N}$.

The $2 n$-th (radial) moments of this distribution are

$$
\begin{aligned}
\mu_{n}^{(M)} & =\left\langle R^{2 n}\right\rangle \\
& =\int R^{2 n} f^{(M)}\left(x_{1}, x_{2}, \cdots, x_{M}\right) \mathrm{d} x_{1} \mathrm{~d} x_{2} \cdots \mathrm{d} x_{M} \\
& =S_{N-M}(1) \frac{S_{M}(1)}{S_{N}(1)} \int_{0}^{1} R^{2 n} R^{M-1}\left(1-R^{2}\right)^{\frac{N-M}{2}-1} \mathrm{~d} R \\
& =\frac{\Gamma(N / 2) \Gamma(n+M / 2)}{\Gamma(M / 2) \Gamma(n+N / 2)} .
\end{aligned}
$$

Explicitly, we obtain

$$
\begin{aligned}
\mu_{0}^{(M)} & =1 \\
\mu_{1}^{(M)} & =\frac{M}{N}, \\
\mu_{2}^{(M)} & =\frac{M+2}{N+2} \cdot \frac{M}{N}, \\
& \vdots \\
\mu_{n}^{(M)} & =\frac{M+2(n-1)}{N+2(n-1)} \cdot \mu(n-1),
\end{aligned}
$$

which yields

$$
\mu_{n+1}^{(M)}<\mu_{n}^{(M)} \quad \text { and } \quad \lim _{N, M \rightarrow \infty} \mu_{n}^{(M)}=\alpha^{n} ;
$$

recall that $1 \leqslant M<N$ holds. In the above limit we keep $\alpha=\frac{M}{N}$ constant. Of particular interest are the mean values $\mu_{1}^{(M)}$ and $\mu_{2}^{(M)}$. On the one hand, we have

$$
\mu_{1}^{(M)}=\int\left(p_{1}+\ldots p_{M}\right) f^{(M)} \mathrm{d} x_{1} \mathrm{~d} x_{2} \cdots \mathrm{d} x_{M} .
$$

This means that $\mu_{1}^{(M)}$ represents the average (total) probability of finding the composite $S+B$ in one of the states $1,2, \ldots M$. On the other hand, the quantity

$$
\sigma=\sqrt{\mu_{2}^{(M)}-\left[\mu_{1}^{(M)}\right]^{2}}=\left\{\frac{2 M(N-M)}{N^{2}(N+2)}\right\}^{\frac{1}{2}}
$$

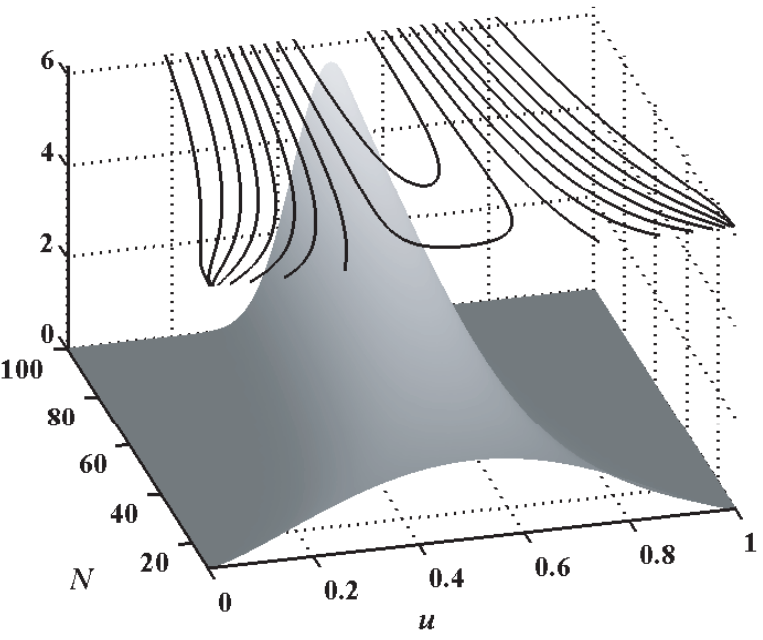

Fig. 1: Density distribution function $F(u)$ as function of $u$ and $N$ for $M=\alpha N$ with $\alpha=0.5$. All quantities are dimensionless. See text for further explanation.

represents the deviation of the aforementioned probability from its average value. From (14) we further find

$$
\frac{\sigma}{\mu_{1}^{(M)}} \rightarrow 0
$$

for $N, M \rightarrow \infty$ with $\alpha=\frac{M}{N}$ constant. This implies that, in the limit of a very large heat bath, for an overwhelming majority of the possible probability distributions on the energy shell $\omega_{S+B}(E)$ the probability of finding $S+B$ in a subset of $A \subset \omega_{S+B}(E)$ is very close to $\frac{M}{N}$, where $M$ is the number of states in $A$. In turn, we can conclude that for the majority of possible probability distributions in the energy shell the system $S$ is described by a probability distribution very close to the canonical one. These considerations are sketched in fig. 1 where the density distribution function

$$
F(u)=\frac{\Gamma(N / 2)}{\Gamma((N-M) / 2) \Gamma(M / 2)} u^{\frac{M}{2}-1}(1-u)^{\frac{(N-M)}{2}-1}
$$

for the probability $u=\sum_{i=1}^{M} p_{i}$ (arising from the uniform distribution on the $N$-dimensional $\left\{x_{1}\right\}$ hyper-sphere) is depicted for different values of $N$ with $\frac{M}{N}=\frac{1}{2}$. As $N$ increases, the density distribution for $u$ becomes more concentrated around the mean value $\langle u\rangle=\frac{M}{N}$.

In sum, we have advanced an argument showing that, even at the purely classical level, the canonical distribution describing a system $S$ weakly interacting with a heat bath $B$ has a degree of inevitability much larger than the one suggested by the standard derivation, which is based on the assumption that the composite $S+B$ is described by a uniform probability distribution in an energy shell; that is, by the microcanonical distribution. When presuming a large heat bath, the overwhelming majority of possible probability distributions on the energy shell 
leads to the canonical ensemble distribution for the system $S$. This conclusion is consistent with the equivalence of ensembles in statistical mechanics, although our present line of thought differs from more conventional arguments. As in the conventional formulation of the equivalence of ensembles, however, our derivation also requires the system under study to be large, i.e. we considered a large heat bath; see [20] for an interesting discussion regarding failure of ensemble equivalence in small systems.

We restricted our present analysis to classical systems with discrete, finite state spaces. Argueably this provides a fair comparison with quantum systems with finitedimensional Hilbert spaces, as considered in [3-5]. The finite state space case enables one to focus on the basic conceptual issues raised by the problem of canonical typicality. On the other hand, classical systems with a discrete state space are of considerable interest in statistical physics; the vast amount of literature devoted, e.g., to the Ising and related models in magnetism, or to lattice models of fluids attests to that. Let us compare our classical arguments with the quantum-mechanical counterpart in more detail. In the quantum case, the microcanonical mixed state for the composite $S+B$ is not a necessary starting point to obtain a canonical density matrix for the system $S$. Indeed, in typical situations, even the most determined quantum states for the composite $S+B$, i.e. pure states, yield a canonical marginal density matrix for $S$. In these cases, the mixedness of the marginal state describing the system $S$ originates in the entanglement between $S$ and the heat bath $B$. In the classical setting, the most determined classical states for the composite $S+B$, corresponding to a complete knowledge of the state of $S+B$, evidently do not yield a canonical distribution for $S$. In this sense, the classical justification for the canonical ensemble is much weaker than the quantum one. However, it is interesting that, even at the classical level, it is not necessary to assume explicitly a microcanonical distribution for $S+B$ in order to obtain a canonical ensemble for $S$ : any typical probability distribution for $S+B$ is sufficient. Of course, it is a minor challenge to find particular probability distributions on the energy shell $\omega_{S+B}(E)$ that do not yield a canonical distribution for $S$. A simple example is given by a probability distribution with one state $k$ with $p_{k}=1$ and the remaining states in the energy shell with zero probability. However, such cases can be regarded as atypical in view of the uniform distribution in probability space given by eq. (6). In fact, the quantummechanical case "suffers" from the same "problem". It is indeed easy to find particular pure states for $S+B$, belonging to the energy shell, that do not lead to a canonical marginal density matrix for $S$. But again, these states can be considered atypical.

Here we discussed the typical features of classical probability distributions for the composite $S+B$ constrained by a fixed total energy. The recent analysis reported in [5] yields a stronger result for the quantum case as it applies for arbitrary constraints. That analysis is based on Levy's lemma which implies that the typical values adopted by a smooth function defined on a high-dimensional hypersphere are very close to the function's average value. One may indeed apply Levy's lemma to the uniform distribution (6) in the classical probability space in order to generalize our present arguments to scenarios involving arbitrary constraints. However, a discussion of this issue is beyond the scope of this letter.

Interestingly, in the classical as well as in the quantummechanical case a "typical" probability distribution for the composite $S+B$ yields a canonical distribution for the system $S$. While in the classical case a probabilistic, i.e. not fully determined, description of $S+B$ is needed, in the quantum case even a maximally determined state of $S+B$, i.e. a pure state, leads to a canonical density matrix for $S$. However, the pure state for $S+B$ implicitly involves probabilistic features too. The analogy between the classical and the quantum-mechanical versions of the high degree of typicality of the canonical ensemble may be regarded as one more instance (along with many others, such as the classical analogues of the quantum no-cloning theorem $[15,16]$; see [14] for a detailed list) of probabilistic or information-related features of quantum mechanics admitting classical counterparts that may be relevant for an eventual clarification of the ontic vs. epistemic debate on the basic meaning of the quantum-mechanical formalism.

$$
* * *
$$

This work was partially supported by the Project FQM-2445 of the Junta de Andalucia (Spain).

\section{REFERENCES}

[1] Feynman R., Statistical Mechanics: A Set of Lectures (Addison-Wesley, Reading, Mass.) 1972.

[2] Thompson C. J., Mathematical Statistical Mechanics (Princeton University Press, Princeton) 1972.

[3] Goldstein S., Lebowitz J. L., Tumulka R. and Zanghi N., Phys. Rev. Lett., 96 (2006) 050403.

[4] Gemmer J., Michel M. and Mahler G., Quantum Thermodynamics (Springer-Verlag, Berlin) 2004.

[5] Popescu S., Short A. J. and Winter A., Nat. Phys., 2 (2006) 754.

[6] Naudts J. and Van der Straeten E., J. Stat. Mech. (2006) P06015.

[7] Lloyd S., Nat. Phys., 2 (2006) 727.

[8] Gorban A., Physica A, 374 (2007) 85.

[9] Gemmer J. and Michel M., Eur. Phys. J. B, 53 (2006) 517.

[10] Gao Y. B. and Sun C. P., Phys. Rev. E, 75 (2007) 011105.

[11] Brody D. C., Hook D. W. and Hughston L. P., Proc. R. Soc. London, Ser. A, 463 (2007) 2021.

[12] Brody D. C., Hook D. W. and Hughston L. P., J. Phys. A, 40 (2007) F503.

[13] Goldstein S., Lebowitz J. L., Tumulka R. and Zanghì N., J. Stat. Phys., 125 (2006) 1193.

[14] Spekkens R. W., Phys. Rev. A, 75 (2007) 032110. 
[15] Daffertshofer A., Plastino A. R. and Plastino A., Phys. Rev. Lett., 88 (2002) 210601.

[16] Plastino A. R. and Daffertshofer A., Phys. Rev. Lett., 93 (2004) 138701.

[17] Plastino A. R. and Plastino A., Phys. Lett. A, 193 (1994) 140.
[18] Bengtsson I. and Zyczkowski K., Geometry of Quantum States: An Introduction to Quantum Entanglement (Cambridge University Press, Cambridge) 2006.

[19] Amari S., Differential Geometrical Methods in Statistics (Springer-Verlag) 1985.

[20] Naudts J., Europhys. Lett., 69 (2005) 719. 